\title{
A Facial Expression Recognition Algorithm Based on Local Binary Pattern and Empirical Mode Decomposition
}

\author{
Hong Yang ${ }^{1,3 *}$ and Deli Zhu ${ }^{2}$ \\ ${ }^{1}$ Institute of Information Science and Technology, Northwestern University, Xian 710127, China \\ ${ }^{2}$ School of Computer and Information Science, Chongqing Normal University, Chongqing 401331, China \\ ${ }^{3}$ Teaching Affairs Office, Chongqing Normal University, Chongqing 401331, China
}

\begin{abstract}
To improve the efficiency of facial expression recognition, this paper puts forward a kind of recognition algorithm based on local binary pattern (LBP) and empirical mode decomposition (EMD). First of all, process the empirical mode decomposition into the preprocessing facial image, and bring forward many a high frequency images instead of the original image; then, divide the sub domain of the high frequency image and obtain the sub domain LBP histogram and full face histogram; finally, identify the expression of the generated LBP histogram. Through the experiment on JAFFE database, it shows that the method is effective for facial expression recognition.
\end{abstract}

Keywords: Facial expression recognition, EMD, LBP.

\section{INTRODUCTION}

Local Binary pattern referred to as LBP, is a kind of effective local description operator based on pixel element [1]. Since it was originally brought up by the University of Oulu in Finland, domestic and foreign scholars on the nature of LBP have done a lot of research, put forward a variety of improved algorithm, and won a lot of achievements in research and application. Literature [2] made a systematic summarization about the LBP algorithm developed in recent years. Based on the study, it is found that LBP algorithm mainly has the following advantages: The algorithm principle is simple, easy to understand; the code complexity is low, which is easy to implement; it is not sensitive to light, which makes it have certain invariance; Part of the improved algorithm has the scale invariance and rotation invariance. LBP algorithm has been applied to many fields, such as image processing, pattern recognition, target detection and tracking, image content retrieval, and computer vision, etc. Due to that LBP has strong texture description ability; it has the natural advantages of the expression analysis and the face recognition [3-4]. Literature [5] put forward LBP feature description and dynamic texture into expression recognition. Based on weighted LBP [6-8] face feature, is combined with the implementation templates for facial expression recognition. Literature [8] incorporated Gabor operator and LBP in facial expression recognition research. Literature [9] combined LBP operator with PCA analysis method for facial expression recognition research. Empirical Mode Decomposition referred to as EMD, is a highly efficient nonlinear no stationary signal Decomposition method. In 1998
Norden huang et al. put forward Empirical Mode Decomposition. In 2014 the EMD method is proposed using for facial expression recognition by Hasimah Ali. This paper combines LBP texture description ability with frequency of the EMD decomposition advantage facial expression recognition, and gives a detailed analysis of results of algorithm.

This paper is organized as follows: the first section, introduction of related research; the second section, LBP and EMD algorithm system description; the third section, the algorithm analysis of experimental results; the fourth section, conclusion.

\section{THE LBP-EMD ALGORITHM}

The LBP-EMD algorithm system mainly includes the following parts: first it is needed to implement noise processing of image identification system, normalization of scale, gray scale normalization and Angle correction operation, and generate the standard picture acceptable to recognition system. The result of the process will directly affect the recognition efficiency. This stage is called "image preprocessing". Second, decompose the image according to a nonlinear signal EMD, obtain image frequency component and allowance, remove the allowance which shows image trend and eliminate part of low frequency components, keep some high frequency components assembled into the EMD high-frequency images, this phase is called 'characteristics processing'; Again, divide the high-frequency image according to a certain size of the number of child windows, generate the child window LBP feature, unite each child window of LBP histogram to form image LBP histogram, this stage is called "feature extraction"; Finally, use the generated LBP histogram for facial expression recognition. (1).

Identification process is as shown in aforementioned Fig. 


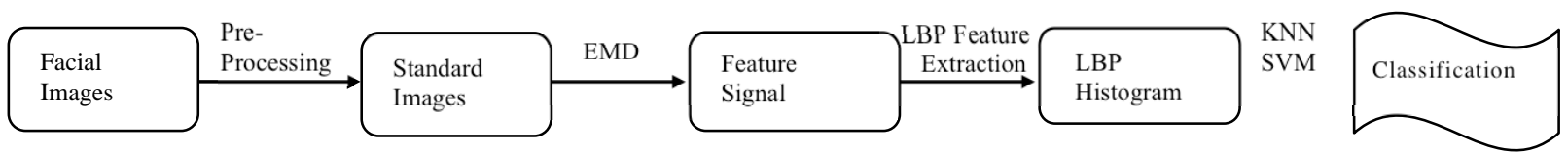

Fig. (1). The facial expression recognition based on LBP-EMD algorithm.

\subsection{LBP Histogram}

Based on the literature [1], the original LBP is a rectangular neighborhood system, consists of the center element and the adjacent 8 elements, such as Fig. (2). Acquire the whole image of LBP expression, that is, the LBP operation of each pixel in the image and connect the generated LBP features. Pixel LBP operation is as follows: take the center pixel value as threshold value, compare the pixels value of each element in the 8 neighborhood with it, when it is greater than or equal to the center pixel of, the pixel values are coded as 1 , less than the code is 0 . Clockwise from top left corner elements connected to form the point of local binary pattern.

\begin{tabular}{|c|c|c|}
\hline 69 & 72 & 94 \\
\hline 26 & 87 & 142 \\
\hline 20 & 98 & 105 \\
\hline
\end{tabular}

\begin{tabular}{|c|c|c|c|}
\hline 0 & 0 & 1 & \multirow{3}{*}{$\begin{array}{l}\text { Binary:00111100 } \\
\text { Decimal:60 }\end{array}$} \\
\hline 0 & 0 & 1 & \\
\hline 0 & 1 & 1 & \\
\hline
\end{tabular}

Fig. (2). The basic principle of LBP operator.

From the above, the pixel values of LBP as the center pixel values are connected to the adjacent pixel point comparison results binary string, called a binary form LBP. Another representation is a decimal method, according to the weighted neighborhood pixel location and, decimal values of LBP is available, and the weighted summation formula is as follows:

$$
\operatorname{LBP}\left(x_{c}, y_{c}\right)=\sum_{i=0}^{7} s\left(g_{i}-g_{c}\right) \times 2^{i}
$$

To overcome the constraints of the original rectangle neighborhood operator, and improve the operation ability of LBP operator on the multi-scale, Ojala et al. extended the basic LBP operator. Main idea is from a square 8 neighborhood extensions to a radius " $\mathrm{r}$ " of the circular neighborhood, participating in the position of the point described by pixel LBP, no matter how many it is, it can go through adaptive change. Fig. (4) is the three basic circular LBP algorithm model, and Fig. (4) is a LBP image effect of the operation.
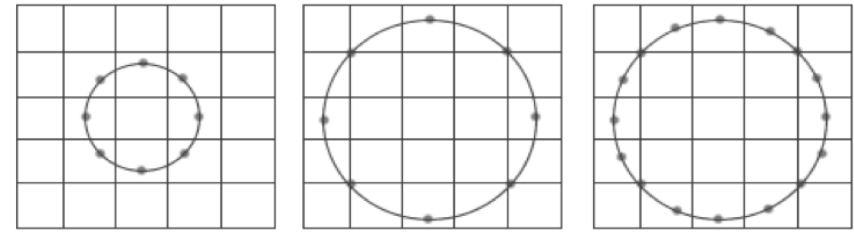

Fig. (3). The principle of extension circular LBP operator.

A pair of gray image for LBP operation can get a pair of LBP image, such as Fig. (4). To obtain local statistical features of the LBP feature maps, first of all, divide the sub do- main in accordance with the relevant provisions of the width and height. Then, get a statistical value of each sub domain LBP values which can generate the histogram and normalize histogram. Finally, connect all of histograms sequentially to form the whole LBP histogram of the image.
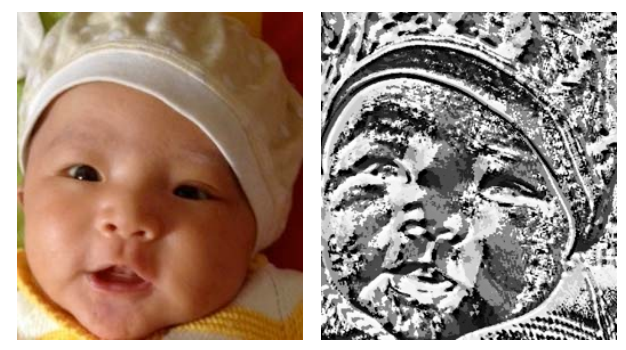

Fig. (4). The image effect based on LBP operator.

\subsection{The EMD Characteristic Processing}

In 1998 Norden huang et al. Put forward empirical mode decomposition (EMD). The method is a kind of self-adaptive analysis method to decompose no stationary nonlinear information, which segregates the signal from high frequency to low frequency by degrees. The final signal can be expressed by the time-frequency component at all levels and the overall trend, which were respectively the intrinsic mode functions and allowance. The EMD decomposition emphasizes on two main aspects: the intrinsic mode functions and process of "filter".

\section{(1) The Intrinsic Mode Function (IMF)}

According to Norden huang, the intrinsic mode function must meet two conditions: (1) in the whole time course, the number of times through zero point and the extreme points equals or differs at most 1 ; (2) in any point on signal, the mean value of upper envelope and lower envelope which were respectively defined by the local maximum values and local minimum value is 0 , namely the signal local symmetry about the timeline.

\section{(2) The "Filter" Process}

The "filter" process is to constantly isolate the signal components in line with features of intrinsic mode function, and separate the remaining components as a new signal process, until no features left which fit the intrinsic mode function but image overall trend. In the process, signal is separated out from high frequency to low frequency. Set one original signal, following a more detailed description of the specific separation process.

The first step is to get the maximum and minimum value of signal $\mathrm{X}(\mathrm{t})$, and then use the cubic spine function fitting into upper and lower envelope line, get the mean $m$ of upper and lower envelop, obtain the difference of $\mathrm{X}(\mathrm{t})$ and $\mathrm{m}_{1}$. 


$$
h_{1}=X(t)-m_{1}
$$

Finally, judge whether meet the IMF's conditions, repeat the above process if not, vice versa became the first order of IMF component (the first high frequency component).

The second step, isolating from, allowance as a new signal is the first step, until the order " $\mathrm{n}$ " allowance be monotonic function and decomposition termination.

$$
\begin{aligned}
& r_{1}=X(t)-C_{1} \\
& r_{n}=r_{n-1}-C_{n}
\end{aligned}
$$

The third step, through the above two steps, the signal is separated in accordance with the frequency, the final signal can be represented as the sum of IMF components and allowance.

$$
X(t)=\sum_{j=1}^{n} C_{j}(t)+r_{n}
$$

By (4) it can be seen that a non-stationary nonlinear original signal was decomposed into the sum of different frequency components. The first separation is the high frequency component; the second is the sub-frequency component, and so forth. The separated IMF component can represent the image of the IMF. One way is to use the isolated independent IMF component, such as Fig. (5); another way is to use the isolated IMF component combination, such as Fig. (6).

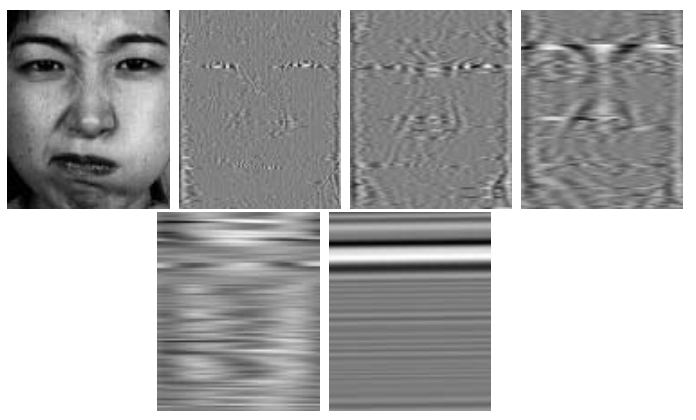

Fig. (5). LBP feature images of the IMF's independent component.

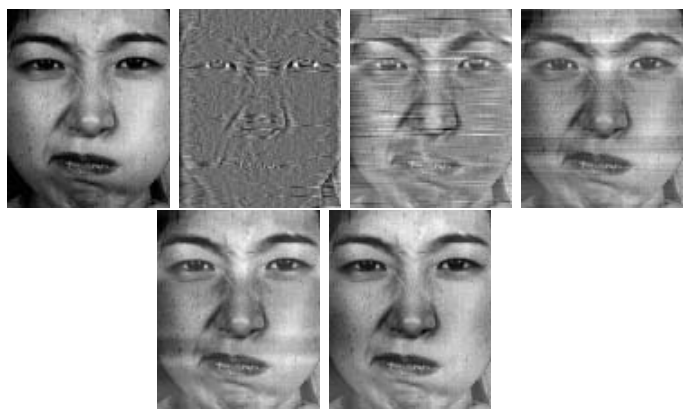

Fig. (6). The effect of IMF.

\subsection{The LBP-EMD Expression Recognition}

The expression database this paper adopts is based on JAFFE database, which is set up by the Japanese ATR, and is all women expressions of 10 people, which includes 213 expression images of seven kinds of expressions. Seven kinds of expressions are: neutral, happy, sad, surprise, anger, disgust, fear, which is all front facial looks. The image size and angle are well adjusted and there is only slight difference in light. First, put the picture database for illumination normalization processing; classify the pictures according to the expression. Some of these images will enter the training set, the rest into the test set. Fig. (7) is for part of happy expressions of the image database.

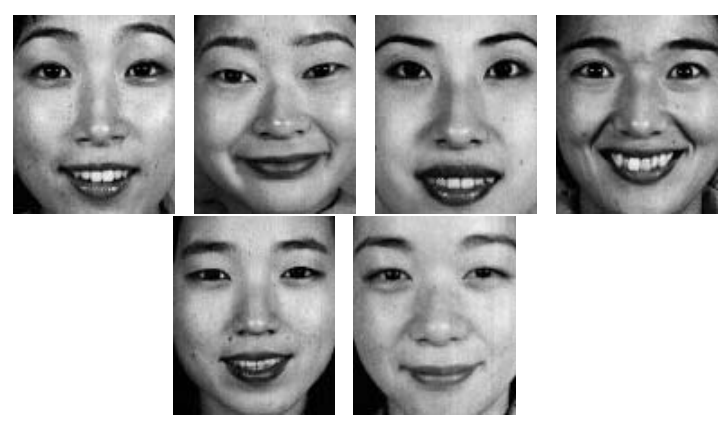

Fig. (7). The standardization images of happiness.

Secondly, extract the image of the component. According to the principle of the EMD algorithm, images of the nonstationary signal can be decomposed into the IMF component and allowance, in which IMF component represents the changes in the image signals of different frequencies, and allowance represents a trend of image signal. For the analysis of the expression, the allowance can be removed. The earlier IMF component can be separated, the better can it reflect the change of the expression, and therefore, making some IMF component combination can approximate table expression changes. Fig. (8) is a happy expression of the image decomposition. Fig. (9) is the fifth IMF component of 7 kinds of expression image contrast.

The last step is based on the EMD combination image of LBP feature recognition. Combine the first 6 IMF components, after that the image synthesis approximately represents the original image, then apply LBP algorithm to composite images and generate LBP image recognition and classification.

\section{EXPERIMENTAL RESULTS AND ANALYSIS}

To test the effectiveness of the expression classification and recognition based on LBP-EMD algorithm, this article adopts the algorithm design based on QT and Open CV, and makes use of the expression database from Japan JAFFE to do grouping experiment. In order to improve the recognition effect, the image is preprocessed and standardized, unified for the specification. Two kinds of experiments are designed, one kind is for facial expression recognition based on LBP method and recognition effect contrast based on EMD - LBP facial expression recognition; Another is to take different amounts of the IMF component EMD - LBP expression to compare the recognition effect. 

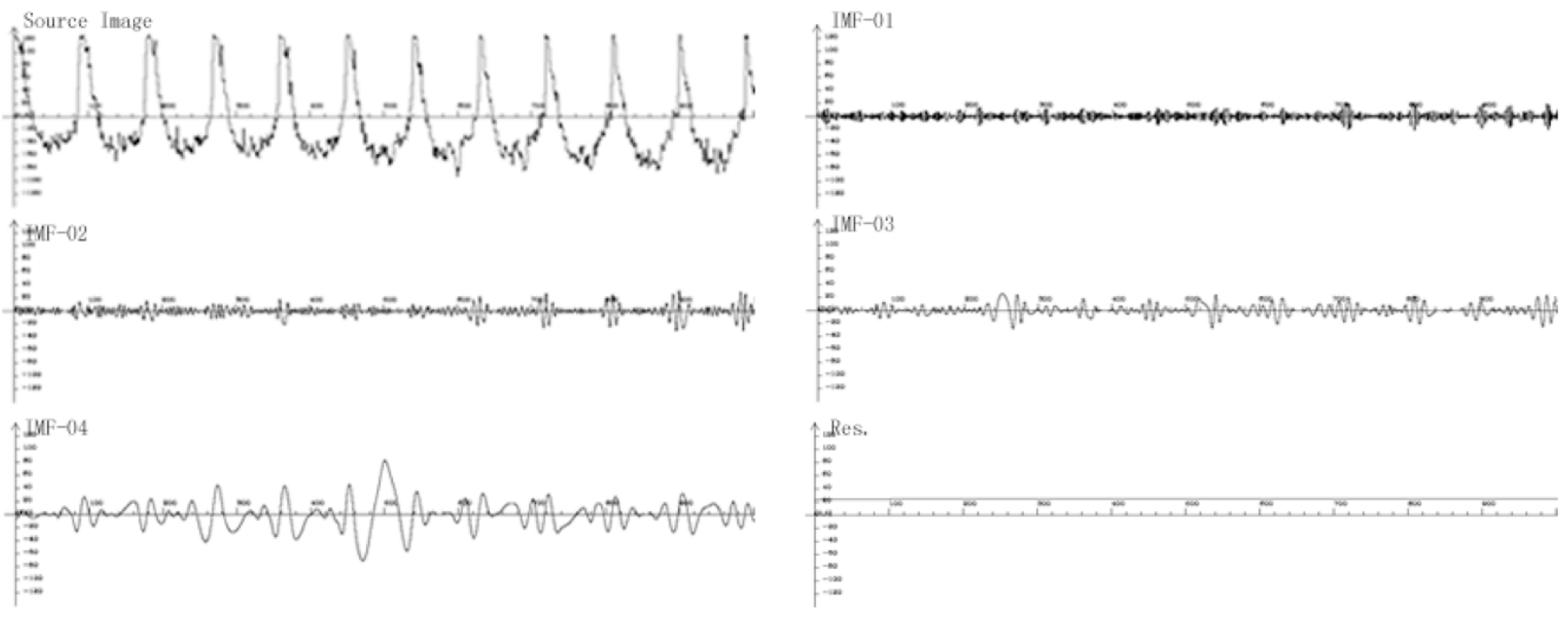

Fig. (8). The "filter" process.
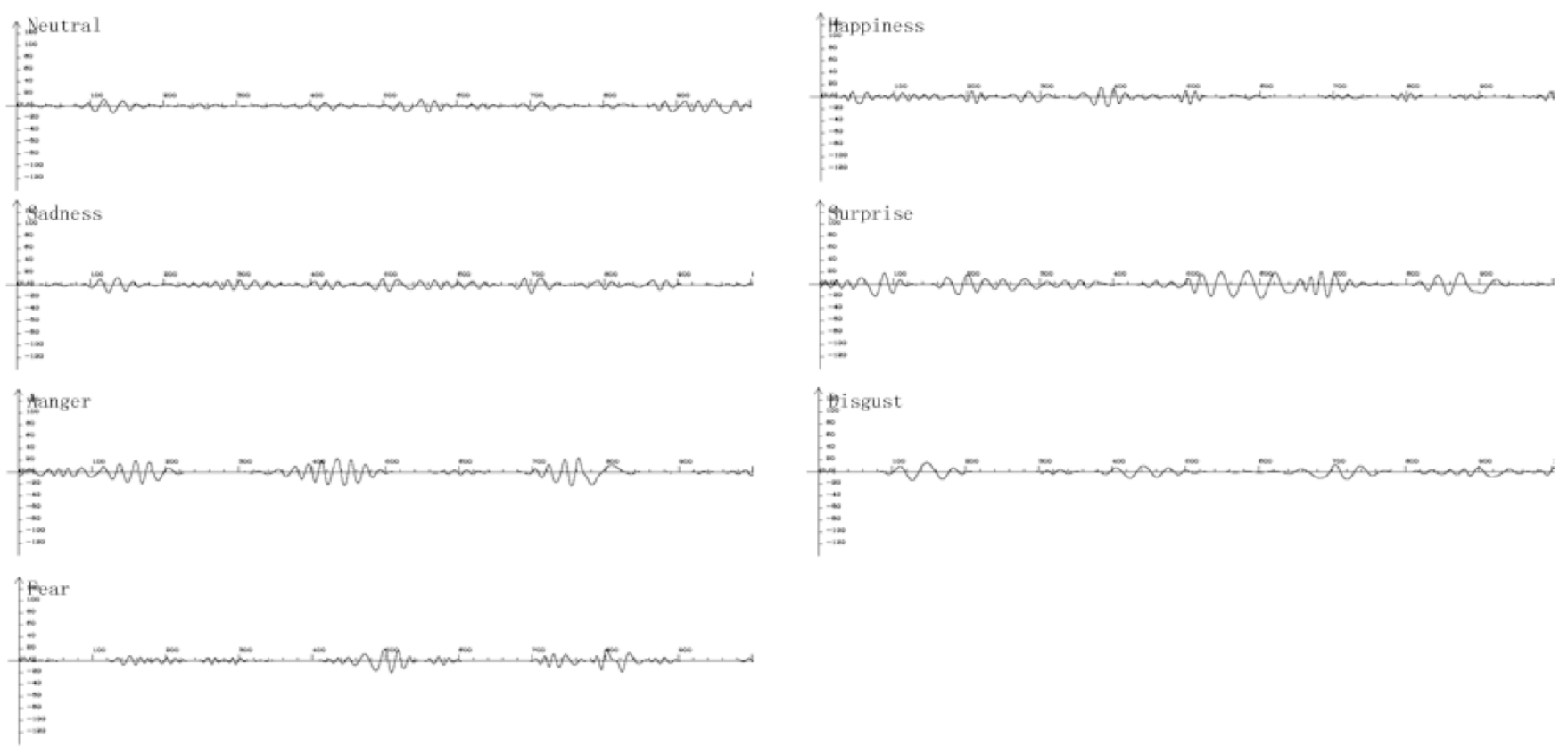

Fig. (9). All kinds of expression contrast.

Fig. (10) is LBP algorithm and the effect comparison chart of the EMD - LBP algorithm. To "neutral, anger, happiness, sadness, and surprise, disgust, fear", the 7 expression categories, identify them respectively using LBP, the EMD LBP algorithm. It can be seen from the effect of the recognition that the identifying effect of EMD-LBP algorithm is overall better than that of LBP algorithm, besides, the EMD - LBP algorithm improves the effect of 3 types of expressions- neutral, sadness, disgust, which are not well operated through LBP algorithm.

Tables 1 to $\mathbf{3}$ contain different IMF components and EMD - LBP effect of facial expression recognition algorithm, in which Table 1 takes the first four IMF components, Table 2 takes the first six IMF components, Table 3 takes 10. Some expression images are employed in

the experiment for the test, which includes 26 "neutral", 26 "anger", 19 "happiness", 19 "sadness", and 26 "surprise", 17 "disgust", 28 "fear". From the three statistical chart, the overall effect is best when taking 6 IMF components, which shows 6 IMF components can basically reflect the change of the face image; the recognition effect of expression "happiness", "disgust" is lower than other expressions.

As was discussed above, conclusion can be drawn as follows: first of all, the EMD - LBP algorithm is effective and feasible. Compared with the pure LBP algorithm, the LBP the EMD algorithm removes image trend, retains the expression of high frequency, combining with LBP operator, can achieve higher recognition efficiency; Second, how to determine the high frequency IMF component quantity. For facial expression recognition, basically taking around 4-10 


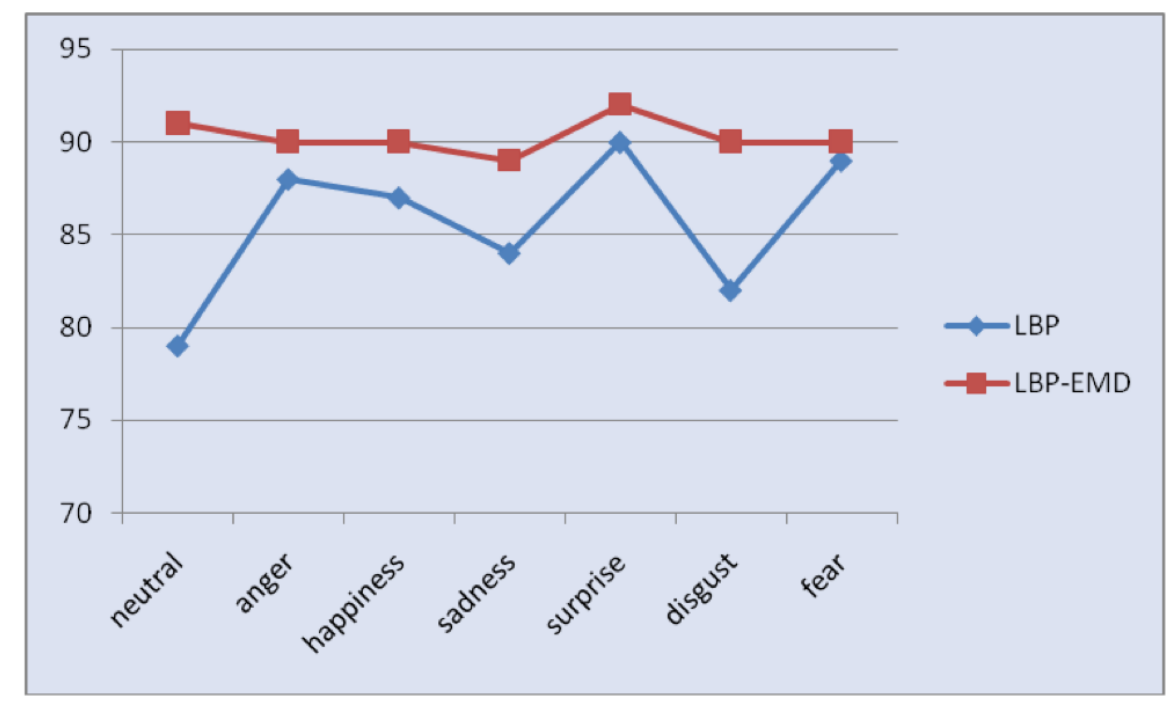

Fig. (10). LBP algorithm and LBP-EMD algorithm effect contrast.

Table 1. The LBP-EMD (IMF1-4) algorithm recognition effect.

\begin{tabular}{|c|c|c|c|c|c|c|c|}
\hline & Neutral & Anger & Happiness & Sadness & Surprise & Disgust & \\
\hline \hline Neutral & 21 & & 2 & 3 & & \\
\hline Anger & 1 & 25 & & & & \\
\hline Happiness & 3 & 1 & 13 & 2 & & 1 \\
\hline Sadness & 1 & 1 & & & 20 & 2 \\
\hline Surprise & 3 & 1 & & & & 16 \\
\hline Disgust & & 2 & & & 2 & 2 \\
\hline Fear & & & & & 24 \\
\hline
\end{tabular}

Table 2. The LBP-EMD (IMF1-6) algorithm recognition effect.

\begin{tabular}{|c|c|c|c|c|c|c|c|}
\hline & Neutral & Anger & Happiness & Sadness & Surprise & Disgust & Fear \\
\hline Neutral & 25 & & & 1 & & & \\
\hline Happiness & & & 16 & 2 & & 1 & \\
\hline Sadness & 1 & & & 17 & & & 1 \\
\hline Disgust & & 2 & & & 1 & 12 & 2 \\
\hline Fear & & & & 1 & & 1 & 26 \\
\hline
\end{tabular}

components can represent the expression of the original image information, about six works best.

\section{CONCLUSION}

Facial expression recognition based on LBP histogram has a certain effect, on this basis, this paper proposed the recognition method based on the combination of EMD and
LBP algorithm, from which, the method eliminates partial information irrelevant to expression through the separation of frequency components, so as to improve the recognition effect. Experiments show that compared with the pure LBP algorithm, the new method has better recognition effect; At the same time, the experimental results show that the extraction of six IMF components or so is enough to indicate changes in facial expressions. 
Table 3. The LBP-EMD (IMF1-10) algorithm recognition effect.

\begin{tabular}{|c|c|c|c|c|c|c|c|}
\hline & Neutral & Anger & Happiness & Sadness & Surprise & Disgust & Fear \\
\hline Neutral & 22 & & & & 3 & & 1 \\
\hline Anger & & 21 & 1 & & 3 & & 1 \\
\hline Happiness & & & 12 & 1 & 5 & & 1 \\
\hline Sadness & 1 & & & 13 & 1 & 1 & 3 \\
\hline Surprise & & & & & 25 & & 1 \\
\hline Disgust & 1 & 1 & & & 3 & 9 & 3 \\
\hline Fear & & & & 1 & 2 & 1 & 24 \\
\hline
\end{tabular}

\section{CONFLICT OF INTEREST}

The author confirms that this article content has no conflict of interest.

\section{ACKNOWLEDGEMENTS}

This research is partially supported by the science and technology projects fund of Chongqing China Grant cstc2013kjrc-qnrc40001 and cstc2013jcyjA80013.

\section{REFERENCES}

[1] T. Ojala, M. Pietikainen, and D. Harwood, "A comparative-study of texture measures with classification based on feature distributions", Pattern Recognition, pp. 51-59, 1996.

[2] L. Liu, Y.X. Xie, Y.M. Wei, and S.Y. Lao, "Survey of Local Binary Pattern method", Journal of Image and Graphics, vol. 19, no. 12, pp. 1696-1720, 2014.

[3] A. Hadid, T. Ahonen, and M. Pietikinen, Handbook of Texture Analysis, London: Imperial College Press, pp. 347-373, 2008.
[4] A. Hadid, "The local binary pattern approach and its applications to face analysis", Proceedings of International Workshops on Image Processing, Theory, Tools and Application, Washington DC: IEEE Computer Society, 2008, pp. 1-9.

[5] G.Y. Zhao and M. Pietika, "Dynamic Texture Recognition Using Local Binary Patterns with an Application to Facial Expressions", IEEE Transactions on pattern analysis and machine intelligence, vol. 29, no. 6, June 2007.

[6] C.F. Shan, S.G. Gong and W.M. Peter, "Robust facial expression recognition using local binary patterns", IEEE International Conference on Image Processing, 2, pp. 370-373, 2005.

[7] R.S. Smith and T. Windeatt, "Facial Expression Detection using Filtered Local Binary Pattern Features with ECOC Classiers and Platt Scaling", JMLR: Workshop and Conference Proceedings 11, 2010, pp. 111-118.

[8] S.M. Lajevardi and Z.M. Hussain, "Facial expression recognition using log-gabor filters and local binary pattern operators", MUSCAT, 15-18, February 2009.

[9] Y. Luo, C.M. Wu and Y. Zhang, "Facial expression recognition based on fusion feature of PCA and LBP with SVM", Optik, 124, pp. 2767-2770, 2013.

(c) Yang and Zhu; Licensee Bentham Open.

This is an open access article licensed under the terms of the Creative Commons Attribution Non-Commercial License (http://creativecommons.org/licenses/by-nc/3.0/) which permits unrestricted, non-commercial use, distribution and reproduction in any medium, provided the work is properly cited. 